\title{
Laser Scattering Measurement of the Electron Density Fluctuations in CHS
}

\author{
Yoshifumi AZUMA, Shunji TSUJI-IIO, Tsuyoshi AKIYAMA ${ }^{1)}$, Kazuo KAWAHATA ${ }^{1)}$, \\ Shoichi OKAMURA ${ }^{1)}$, Shigeki OKAJIMA ${ }^{2)}$, Kazuya NAKAYAMA ${ }^{2)}$, Ryuichi SHIMADA, \\ Hiroaki TSUTSUI, Kenji HIGUCHI and CHS experimental group ${ }^{1)}$ \\ Tokyo Institute of Technology, Ookayama, Meguro-ku, Tokyo 152-8550, Japan \\ ${ }^{1)}$ National Institute for Fusion Science, Toki, Gifu 509-5292, Japan \\ 2) Chubu University, Matsumoto-cho, Kasugai-shi, Aichi 487-8501, Japan
}

(Received 4 December 2006 / Accepted 21 May 2007)

\begin{abstract}
For measurement of electron density fluctuations (up to $1 \mathrm{MHz}$ ), an $\mathrm{HCN}$ laser scattering measurement system with a super rotating grating was developed on CHS. When we measured electron density fluctuations with $k r=5.4 \mathrm{~cm}^{-1}$ in a plasma edge region at the outer side of torus, fluctuation spectra which have harmonic components up to the fifth were observed. The fundamental frequency was less than about $60 \mathrm{kHz}$, which changed during the discharge. The change in frequency correlated best with the plasma stored energy divided by the line averaged density. An edge transport barrier (ETB), which can improve particle transport in the edge region, has been observed in CHS and the measured fluctuation amplitude also decreases. The fluctuations decreased in about $5 \mathrm{~ms}$, and the decrease of the fluctuations preceded by $3 \mathrm{~ms}$ to the drop of $\mathrm{H} \alpha$ emission signal.
\end{abstract}

(C) 2007 The Japan Society of Plasma Science and Nuclear Fusion Research

Keywords: compact helical system, electron density fluctuation, laser scattering measurement, heterodyne technique, HCN laser, harmonic components of fluctuation spectra, ETB formation

DOI: $10.1585 /$ pfr.2.S1109

\section{Inroduction}

Anomalous transport plays a dominant role in plasma confinement in magnetic confinement fusion devices. Since fluctuations cause anomalous transport and degrade plasma confinement, the understanding of physical mechanisms for fluctuations is one of the important issues towards realization of fusion reactors [1]. In this study, it was examined specifically about electron density fluctuations in the Compact Helical System (CHS).

Although there are some methods for measurement of electron density fluctuations, an electromagnetic wave scattering method was adopted for the measurement $[2,3]$. This method is excellent in terms of the measurement accuracy of wave number. The plasma measurement which used a far infrared laser beam has advantages that it can avoid the cutoff by the plasma because the frequency of the laser beam is sufficiently higher than the electron plasma frequency and that the local measurement of plasma becomes possible because effect of the plasma refraction becomes little.

An edge transport barrier (ETB), which can improve particle transport in the edge region, has been observed in CHS $[4,5]$. Therefore it is suitable for discussing how fluctuations influence confinement of plasma.

\section{Optical Systems}

Figure 1 is a schematic diagram of incident and scattered beams in a plasma. The frequency and the amplitude of electron density fluctuations are extracted from the spectrum of scattered beam. With the Bragg relation $k=2 k_{i} \sin \left(\theta_{s} / 2\right)$, the scattering angle $\theta_{s}$ is related to the wave number of electron density fluctuations $k$, where $k_{i}$ is the wave number of incident beam. Hence, the wavenumber of fluctuations can be selected from the line of sight of detection optics.

The measurement system uses a hydrogen cyanide (HCN) laser (wavelength: $337 \mu \mathrm{m}$ ) as the light source $[6,7]$. Operational output power of HCN laser is about $100 \mathrm{~mW}$ (maximum of about $500 \mathrm{~mW}$ ) for stabilities of the frequency and the output power. The wavelength of the $\mathrm{HCN}$ laser is appropriate for the scattering measurement

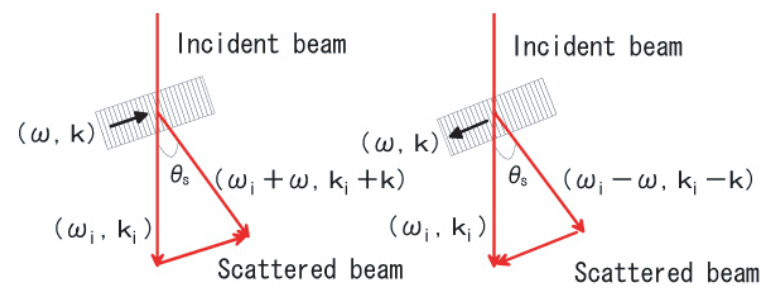

Fig. 1 Schematic diagram of incident and scattered beams in plasma. 
on medium-sized fusion devices such as CHS. The scattering of the electromagnetic wave is attributed to two types of motion of electron in plasma [8]. The one is a thermally individual motion. The other is collective motion of electrons, which is caused by drag by movement of ions or plasma instabilities. The Salpater parameter $\alpha=1 / k \lambda_{D}$ is useful to determine which component is dominant, where $\lambda_{D}$ is the Debye length of the plasma. When $\alpha>1$, information on collective motion of electrons is obtained. The frequency and wave number of fluctuations can be evaluated from the frequency shift and scattering angle of scattered beam. In a fusion plasma the wavelength of $\mathrm{HCN}$ laser satisfies $\alpha>1$. In the case of shorter wavelength, the scattering angle is so small that the length of scattering volume along beam axis becomes long and a long optical path is necessary to separate the scattered beam from the incident beam. On the other hand, beam deviation due to plasma refraction also becomes a serious problem for long wavelengths such as millimeter waves. From above two points of views, the $\mathrm{HCN}$ laser is appropriate for the scattering measurement on CHS.

The measurement system adopted heterodyne technique. It can distinguish the propagation direction of fluctuations, which is a useful hint to identity the driving force. As for the heterodyne measurement, the frequency of the local beam is required to be different from that of the probe beam. There are two methods used often to shift the frequency. One is to use a rotating grating [9] which causes the Doppler shift and the other is to use a twin laser system [9]. In a method which uses a rotating grating, system is simple and the frequency is stable. However, the obtained beat frequency, which determines the maximum of the time resolution, is low (usually less than $100 \mathrm{kHz}$ ) due to the limitation of the mechanical rotation speed. On the other hand, in a twin laser system the cavity length of one laser is controlled to have a frequency difference from the other. This method can produce a beat frequency of higher than $1 \mathrm{MHz}$. However, the laser system tends to be so complicated. In this study a Super Rotating Grating (SRG) [10] is adopted, not being the conventional rotating grating. The SRG makes it possible to realize high-frequency beat signal up to $1.45 \mathrm{MHz}$, which is comparable to that of the twin laser system. This enables us to measure fluctuations in a wide range of frequency. At this measurement the beat frequency is $1 \mathrm{MHz}$. Therefore the frequency range of measurable fluctuation is $\pm 1 \mathrm{MHz}$. The beat frequency is stable and the variations are less than $\pm 0.2 \mathrm{kHz}$.

Schematic layout of the scattering measurement system with the HCN laser and the SRG installed on CHS is shown in Fig. 2. The optical path length from the HCN laser to the input window is $18 \mathrm{~m}$. The laser beam is transmitted with waveguides (inner diameter of $47 \mathrm{~mm}$ ) made of acrylic resin to CHS. The total transmission efficiency is about $80 \%$ (path length of waveguides is about $15 \mathrm{~m}$ ). In order to make the beam transmission simple the SRG should be placed near the optical system for detection, if (a)

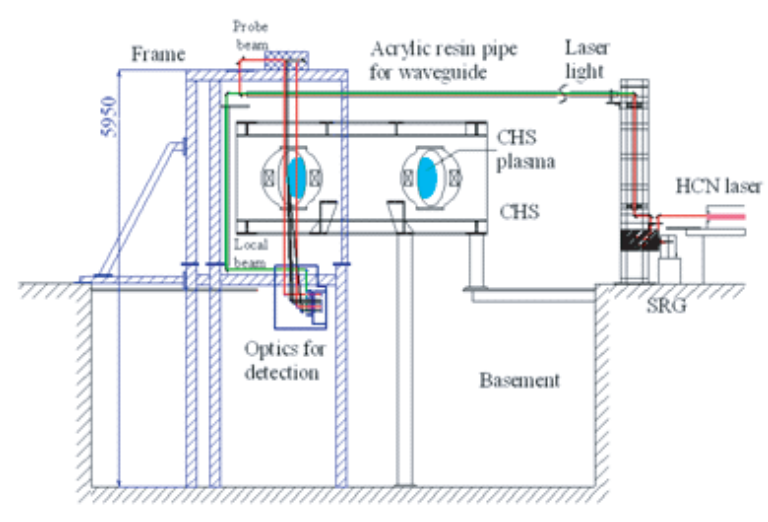

(b)

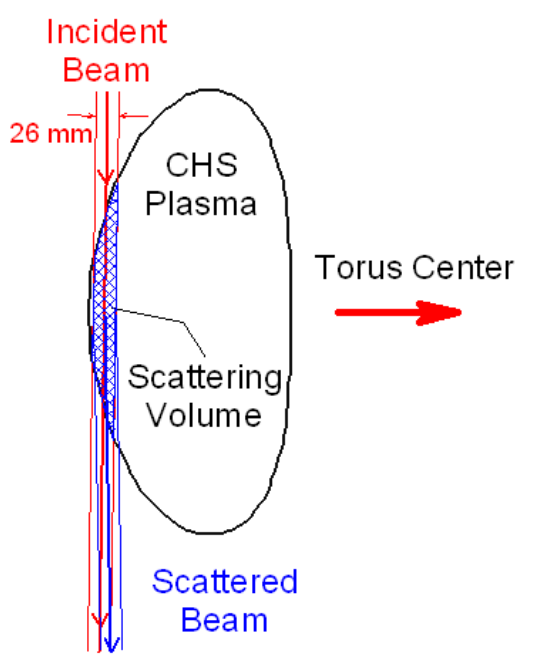

Fig. 2 (a) Elevation view of the HCN laser scattering system on CHS (b) The poloidal cross section of the CHS plasma and the location of the scattering volume in CHS plasma.

possible. However, it was placed far from CHS because stray magnetic fields from CHS are not negligible and any disturbances on the rotation of the grating, which is magnetically suspended, should be avoided. The probe beam is injected from the upper part of CHS. The beam width of the probe beam is $26 \mathrm{~mm}$ on plasma equatorial plane as shown in Fig. 2 (b) the location of the scattering volume is a plasma edge region at the outer side of torus, where the electron density gradient is large and the transport barrier is formed. The optics of heterodyne detection are placed at a lower space of CHS. Detectors are Schottky barrier diodes. After passing through an analog high-pass filter and an amplifier, $1 \mathrm{MHz}$ beat signals of the probe and reference are digitized at a frequency of $5 \mathrm{MHz}$. There are three detectors for scattering measurement. Three detectors can detect fluctuations of wave number $k r=5.4 \mathrm{~cm}^{-1}, k_{\mathrm{r}}=9.8 \mathrm{~cm}^{-1}$, $k_{\mathrm{r}}=20.3 \mathrm{~cm}^{-1}$, respectively. However, in this study only $k_{\mathrm{r}}=5.4 \mathrm{~cm}^{-1}$ which has the strongest fluctuation spectral intensity was investigated. The frame was installed on the floor of the basement without touching CHS to avoid mechanical vibrations. Since this optical system is in combi- 
nation with an interferometer for the densimetry, optics for detection is shared with the interferometer system.

\section{Experimental Results}

\subsection{Analysis of fluctuations with harmonic components}

Figure 3 shows the time evolution of operational and plasma parameters and the frequency spectra. Plus and minus frequency corresponds to the inward and outward direction of propagation, respectively. The magnetic field strength at the magnetic axis position, $R_{\mathrm{ax}}=92.1 \mathrm{~cm}$, is $B_{\mathrm{ax}}=1.97 \mathrm{~T}$. In the inward direction, there are turbulent fluctuations up to $200 \mathrm{kHz}$. Sharp fluctuation peaks, which have harmonic components up to the fifth, were observed. The fluctuations propagate only in the outward direction and the amplitude of the second component is always the largest among harmonic components. Frequency of harmonic components is shifted largely whereas the intensity was nearly constant in this shot, and increase rapidly with the increase in the stored energy at the beginning of the discharge. While the electron density keeps increasing, the stored energy saturates after $t=0.06 \mathrm{~s}$. This means that the temperature starts to decrease. In that phase the frequency also starts to decrease and the maximum fundamental frequency is about $60 \mathrm{kHz}$ in this discharge. After the application of ECH power from 98 to $103 \mathrm{~ms}$, the frequency of fluctuations appears to increase slightly. After $t=0.11 \mathrm{~s}$ stored energy starts to decrease. In this phase there are intensive turbulent fluctuations up to $50 \mathrm{kHz}$ in any directions.

Figure 4 shows a result of parameter dependence analysis of the frequency of the second harmonic component. It is found that the frequency of harmonics correlates with the plasma stored energy divided by the line averaged density, $W_{\mathrm{p}} / n_{\mathrm{e}}$, which is equivalent to the average temperature. The frequency rises as $W_{\mathrm{p}} / n_{\mathrm{e}}$ value increases. Normally, the information about the local temperature and the density are necessary to be used for the analysis. However, it is difficult to insert Langmuir probes in the scattering measurement position because of a limitation of port access, and there is no electron temperature information because of no ECE data at the position of the scattering volume. The Thomson scattering data are too limited to analyze. From these reasons, it was evaluated with the use of the value which is equivalent to the temperature.

\subsection{Fluctuations in ETB formation}

Figure 5 shows time evolutions of operational and plasma parameters and the frequency spectra in the case of an ETB-formed plasma. After injections of two NBs, the $\mathrm{H} \alpha$ emission signal, which roughly indicates information on the recycling particle flux to the plasma, decreased spontaneously at $t=0.053 \mathrm{~s}$. Then the density gradient in the plasma edge region becomes steeper and the edge transport barrier is thought to be formed [11]. Frequencies
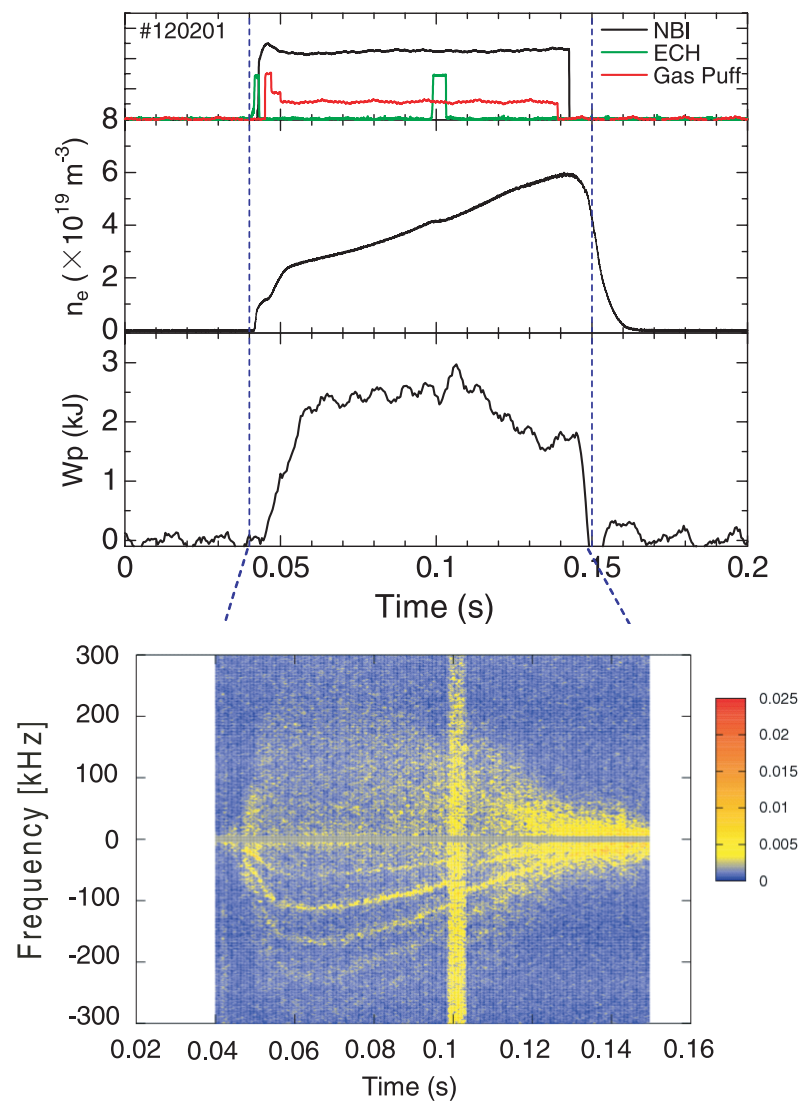

Fig. 3 Time evolution of operational and plasma parameters and frequency spectra whose wave number component is $k_{\mathrm{r}}=$ $5.4 \mathrm{~cm}^{-1}$. Plus and minus frequencies correspond to the inward and outward propagation directions.

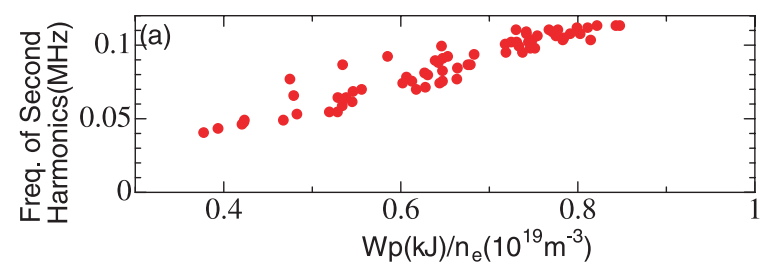

Fig. 4 Relation of freq. of second harmonics to the $W_{\mathrm{p}} / n_{\mathrm{e}}$.

of fluctuations with a wavenumber of $5.4 \mathrm{~cm}^{-1}$ increased up to $\pm 200 \mathrm{kHz}$ after NB injections. However, they were suppressed at the transition. The condition of the low fluctuations level continues until about $t=0.06 \mathrm{~s}$ and then fluctuations with a frequency up to about $10 \mathrm{kHz}$ increase as the density rises. In order to clarify the timing and the time scale of the decrease of fluctuations, spectrum integration in the frequency was computed. This integration value is proportional to the square of the fluctuation amplitude. Figure 6 shows the time evolutions of the integration value and the $\mathrm{H} \alpha$ emission signal at the ETB formation. This figure indicates that the decrease of the fluctuations is earlier by about $3 \mathrm{~ms}$ than $\mathrm{H} \alpha$ drop, and the time scale of the decrease in the integration value $(3-5 \mathrm{~ms})$ is longer than that of the $\mathrm{H} \alpha$ emission signal $(<1 \mathrm{~ms})$. 


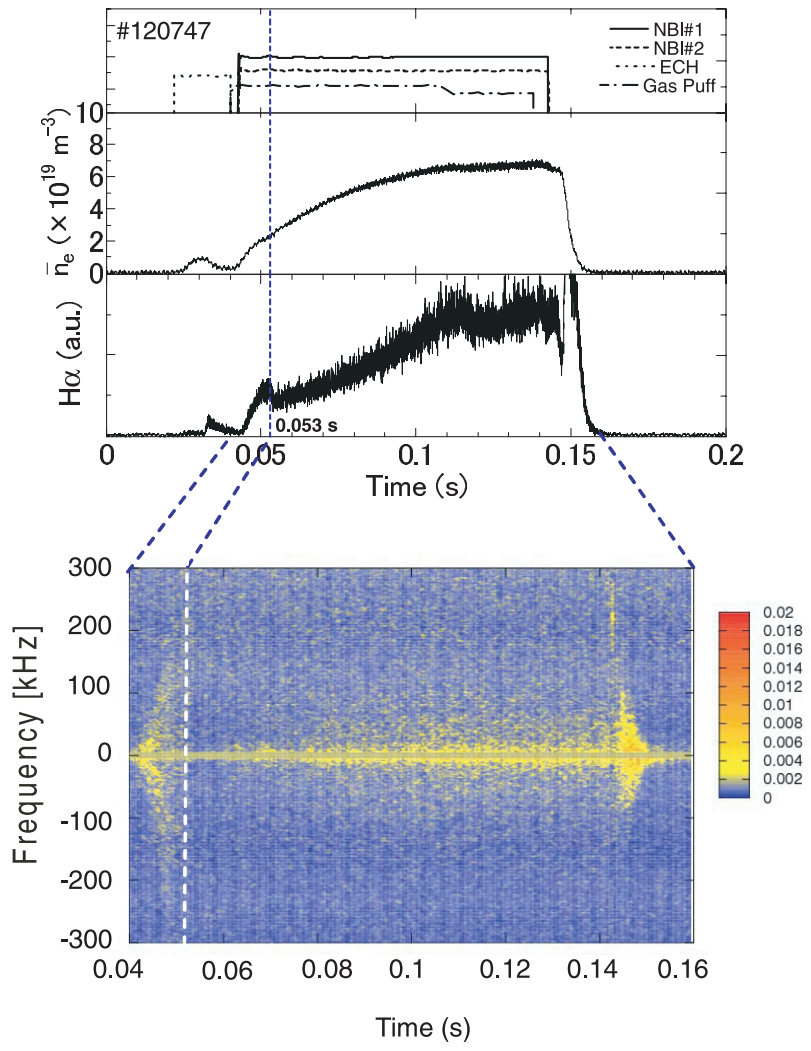

Fig. 5 Time evolution of operational and plasma parameters and frequency spectra whose wave number component is $k_{\mathrm{r}}=$ $5.4 \mathrm{~cm}^{-1}$ in a shot with ETB formation.

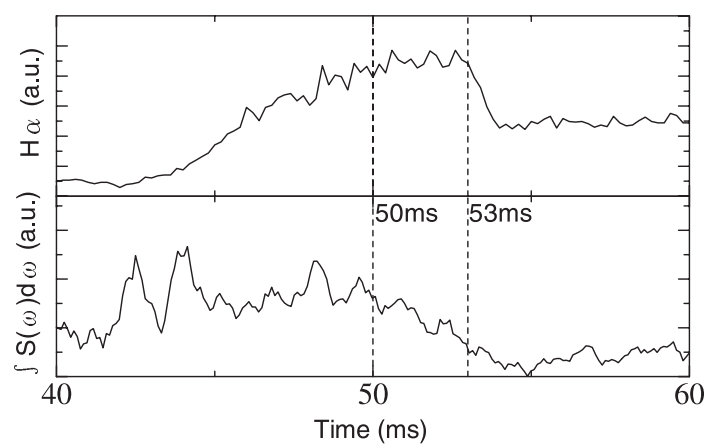

Fig. 6 Time evolutions of the $\mathrm{H} \alpha$ emission signal and a spectrum integration across the frequency in ETB formation.

\section{Discussion}

It has not been clarified what causes the fluctuations with harmonics and why they have harmonic components. One of the fluctuations which have the similar frequency range are toroidal Alfvén eigenmodes (TAEs) [12], which are excited by high energy ions. However, the observed density range in CHS is usually less than about $3 \times 10^{19} \mathrm{~m}^{-3}$ [12] and it is different from that where the fluctuations with harmonic components are observed. Hence, they do not seem to be usual TAEs. The dependence of the frequency on the temperature should be a key. Although geodesic acoustic mode (GAM), whose frequency is a proportional to the square root of the ion and the electron tempera- ture [13], is a plausible candidate for this, the identification is left for future work.

The scattering measurement and the reflectmetry on DIII-D, show a decrease in the density fluctuations approximately at the same time with a sudden drop in $\mathrm{D} \alpha$ emission [14]. On the contrary, the decrease in fluctuations precedes the $\mathrm{H} \alpha$ drop in CHS. Although the potential was not measured in the discharge, it is reported that the change in the potential precedes the $\mathrm{H} \alpha$ drop by several $\mathrm{ms}$ in other discharges [15]. It is possible to think that the decrease in the density fluctuations is related to this. As for the time scale of the decrease in the fluctuations, fluctuations in DIII-D decrease at the time $(20-40 \mu \mathrm{s})$ which is considerably shorter than the energy confinement time. On the other hand, fluctuations in CHS decrease in time comparable to the energy confinement time.

\section{Summary}

Following two were shown by the scattering measurement of the electron density fluctuations in CHS.

1) Sharp fluctuation peaks, which have harmonic components up to the fifth, were observed. The frequency of harmonic components correlates with average temperature defined as the plasma stored energy divided by line average density. The frequency increased with $W_{\mathrm{p}} / n_{\mathrm{e}}$.

2) Electron density fluctuations were observed to be suppressed at ETB formation. The decrease in the fluctuations preceded by about $3 \mathrm{~ms}$ to the drop of $\mathrm{H} \alpha$ emission signal. The time scale of the decrease is comparable to the energy confinement time.

\section{Acknowledgement}

This work is supported by funds of NIFS03KZPD004, NIFS06KZPH001 and NIFS05 ULPD603.

[1] A.J. Wootton et al., Phys. Fluids B 2, 2879 (1990).

[2] D.L. Brower et al., Rev. Sci. Instrum. 61, 3019 (1990).

[3] E. Mazzucato et al., Phys. Plasmas 10, 753 (2003).

[4] S. Okamura et al., J. Plasma Fusion Res. 79, 977 (2003).

[5] S. Okamura et al., Plasma Phys. Control. Fusion 46, A113 (2004).

[6] S. Okajima et al., Conference digest of 11th Int. Conf. on IR and MM Waves (Pisa) 249 (1986).

[7] S. Okajima, et al., InfraredPhys. 29, 331 (1989).

[8] D.L. Brower et al., in Topics in Millmeter wave Technology (Academic Press), Vol.2, pp.83-172.

[9] D. Veron, in Infrared and Millimeter waves (Academic Press), Vol.2, pp.67-135

[10] T. Maekawa et al., Rev. Sci. Instrum. 62, 304 (1991).

[11] S. Okamura et al., Nucl. Fusion 45, 863 (2005).

[12] K. Toi et al., Nucl. Fusion 40, 1349 (2000).

[13] P.H. Diamond et al., Plasma Phys. Control. Fusion 47, R35 (2005).

[14] R.A. Moyer et al., Phys. Plasmas 2, 2397 (1995).

[15] Y. Nagashima et al., Plasma and Fusion Res. 1, 041 (2006). 